\title{
Development of magnetic solid-phase extraction coupled with dispersive liquid-liquid microextraction method for the simultaneous determination of biphenyl and biphenyl oxide in water samples
}

\author{
Arezoo Hassan Noori ${ }^{1}$, Mohammad Rezaee ${ }^{2}$, Maryam Kazemipour ${ }^{1}$ and Hossein Ali Mashayekhi ${ }^{3 *}$ \\ 'Department of Chemistry, Kerman Branch, Islamic Azad University, Kerman, Iran \\ Materials and Nuclear Fuel Research School, Nuclear Science \& Technology Research Institute, \\ Atomic Energy Organization of Iran, P.O. Box 14395-836, Tehran, Iran \\ ${ }^{3}$ Department of Chemistry, Tonekabon Branch, Islamic Azad University, Tonekabon, Iran
}

Received: 22 August 2016; accepted: 27 September 2016

\begin{abstract}
A new and sensitive method, termed magnetic solid phase extraction combined with dispersive liquid-liquid microextracton (MSPE-DLLME), has been developed for the simultaneous determination of biphenyl and biphenyl oxide in water samples. Different parameters influencing the extraction efficiency, including the amount of sorbent, sorption time, type of elution solvent and its volume, type of extraction solvent and its volume, and elution time were optimized. The calibration curves were linear in the range of $0.5-100 \mu \mathrm{g} / \mathrm{L}$ for both of them. The limits of detection (LODs) were achieved, $0.03 \mu \mathrm{g} / \mathrm{L}$ for biphenyl and $0.07 \mu \mathrm{g} / \mathrm{L}$ for biphenyl oxide, respectively. Ultimately, the applicability of the method was successfully confirmed by the extraction and determination of biphenyl and biphenyl oxide in sea, river, tap, and water well.
\end{abstract}

Keywords: Magnetic solid-phase extraction, dispersive liquid-liquid microextraction, biphenyl, biphenyl oxide, gas chromatography

\section{Introduction}

Biphenyl is used as dry carrier for polyesters, feedstock, especially in the production of alkyl biphenyls, and a citrus fruit impregnated wrapping material to reduce spoilage. Biphenyl is also an intermediate for the production of a host of other organic compound, such as emulsifier, optical brighteners, crop protection products, and plastics. Biphenyl oxide (BPO) is used in the production of emulsifiers, surfactant, and textile dye labeled as a chemically reacted intermediate. The main application of this mixture is heat transfer in the distillation tower and fatty acid production liner. This liquid has an optimum heat coefficient with maximum service temperature up to $400{ }^{\circ} \mathrm{C}$. There are many problems in fatty acid distillation tower, i.e., this liquid leaks from stocks to environment. The mixture is very harmful for human and ecological system [1]. Workers exposed to these toxins experience nausea, vomiting, and irritation of the respiratory tract and later result in damage to the liver and nervous system [2]. Toxins enter the aquatic environment through waste effluents from textile mills, industrial processes, and leaking heat exchangers [3, 4]. Conventional methods for analysis of this liquid are liquid-liquid extraction and gas chromatographic analysis, which are very time consuming and use large amount of organic solvent. Reported method for measurement of biphenyl and biphenyl oxide are very limited, and in all of them, solution is directly injected into the instruments: (1) direct injection to gas chromatograph equipped with flame ionization detector (GC-FID) with limits of detection of $5 \mathrm{mg} / \mathrm{L}$ [5], (2) direct injection to gas chromatograph equipped with a mass spectrometer detector (GC-MS) with limits of detection of $10 \mu \mathrm{g} / \mathrm{L}$ [6], and (3) direct injection to high-performance

*Author for correspondence: chem.mashayekhi@toniau.ac.ir; tel.: +98-1924271105; fax: +98-192-4274409. liquid chromatograph equipped with a fluorescence detector (HPLC-FLD) with limits of quantitation of $0.1 \mu \mathrm{g} / \mathrm{mL}$ [7].

Sample preparation prior to instrumental analysis is one of the most crucial steps in the overall analytical process. Generally, liquid-liquid extraction (LLE) and solid-phase extraction (SPE) are considered as the most commonly used techniques for the preconcentration of compounds from various samples [8]. SPE is a well-established method for the preconcentration and isolation of various analytes from different matrices. In SPE, selection of an appropriate sorbent is an important strategy in the elaboration of analytical procedure, and nano-sorbents have proved to be especially effective because of their high specific surface areas, highly active surface sites, and the absence of internal diffusion resistance in the separation process [9].

Magnetic nanoparticles (MNPs) have gained more attention in analytical field for trace analysis and speciation research owing to their susceptibility for bulk solution suspension, easy control, and fast magnetic separation under an external magnetic field [10, 11]. MNPs, such as $\mathrm{Fe}_{3} \mathrm{O}_{4}$, are good candidates for magnetic carrier technology considering the following main advantages: (1) MNPs can be produced in large quantity using a simple method, (2) it can be expected that their sorption capacity is high due to their large surface area, (3) they have strong magnetic properties and low toxicity $[12,13]$, and (4) these particles are super paramagnetic, which means metal-loaded sorbent can be easily separated from the treated water via an external magnetic field. However, the drawbacks of utilizing MNPs for sample preparation are their low selectivity toward target analytes, low stability in strong acidic aqueous media, and low dispersibility in various sample matrices. Therefore, the modification of MNPs with suitable coating has been proven to be one of the most efficient approaches. There has been an increasing interest in establishing new coating materials for MSPE $[14,15]$. In magnetic solid phase extraction

This is an open-access article distributed under the terms of the Creative Commons Attribution-NonCommercial 4.0 International License (https://creativecommons.org/licenses/by-nc/4.0/), which permits unrestricted use, distribution, and reproduction in any medium for non-commercial purposes, provided the original author and source are credited, a link to the CC License is provided, and changes - if any - are indicated. 
(MSPE), achieving high extraction efficiency and recovery might be due to the specific surface area of MNPs, while the whole procedure is rapid, inexpensive, and easy to handle [16-18].

Among the applications of nanocomposites in various fields, magnetic nanocomposites produced by functionalizing polymers with magnetic nanomaterials are becoming popular. The incorporation of both magnetism and polymeric features throughout the nanocomposite structure lead to great potentials in various applications. So far, different magnetic nanocomposites have been synthesized [19], in which among them, magnetic iron oxide (e.g., maghemite $\gamma-\mathrm{Fe}_{2} \mathrm{O}_{3}$ or magnetite $\mathrm{Fe}_{3} \mathrm{O}_{4}$ ) nanoparticles have attracted tremendous attentions due to their low toxicity, stability, and biocompatibility in the physiological environment [20-22]. The extraction and adsorption capacity of $\mathrm{Fe}_{3} \mathrm{O}_{4}$ nanoparticles can be improved by coating and/or functionalizing their surface [23]. Among different types of coating sorbents used for the extraction of organic analytes, composite of conductive polymers due to their multifunctional properties, including hydrophobicity, acid-base character, $\pi-\pi$ interaction, polar functional groups, ion exchange property, hydrogen bonding, and electroactivity are quite prominent [24, 25]. Polypyrrole (PPy) is a conducting polymer with unique properties, namely, high electrical conductivity, relatively good environmental stability, nontoxicity, relatively low cost, and ease of preparation, which are favorable for various types of application in batteries, sensors, supercapacitor, microwave shielding, hybrid material, and as adsorbents [26, 27]. Also, polypyrrole was coated on fused silica capillary's inner surface (GC precolumn) by chemical polymerization. This coated capillary was used successfully for automated in-tube solid-phase microextraction (SPME) [28]. Direct electrochemical deposition of polyaniline-polypyrrole blend coating on the surface of stainless steel wire was used for solid-phase microextraction [29]. Polypyrrole-coated magnetic particles for micro solid-phase extraction were used [30].

Dispersive liquid-liquid microextraction (DLLME) was introduced by Rezaee et al. in 2006 [31]. In this method, an appropriate mixture of extraction and disperser solvents is used. This method has attracted a lot of attention due to its advantages, such as short extraction time, low consumption of organic solvent, and simplicity [32-34].

In this study, polypyrrole as a conductive polymer was coated on the surface of $\mathrm{Fe}_{3} \mathrm{O}_{4}$ nanoparticles. The analytes were extracted from the water samples using magnetic solid-phase extraction (MSPE), followed by dispersive liquid-liquid microextraction. The effect of principle factors, including sorbent amount, extraction time, type of elution solvent, volume of elution solvent, type of extraction solvent, volume of extraction solvent, and desorption time, were studied.

\section{Experimental}

Chemical and Reagents. Biphenyl and biphenyl oxide were provided by Paksan Company (Tehran, Iran). Carbon tetrachloride, tetrachloroethylene, chloroform, chlorobenzene, acetone, acetonitrile, ethanol, and methanol were obtained from E. Merck (Darmstadt, Germany). Double distilled water was used for the preparation of aqueous solutions. Stock standard solutions of the analytes were prepared by the dissolution of each analyte in methanol, having a concentration of $100 \mathrm{mg} / \mathrm{L}$. Fresh standard solutions were prepared by diluting the standard solution of the analytes with deionized water of required concentration. Ferric chloride, ferrous chloride, sodium hydroxide, reagent-grade $\mathrm{NaCl}$, and polypyrrole were purchased from Merck.

Instrumentation. The chromatographic analysis was carried out on an Agilent GC-7890 system equipped with a split/splitless injector system and a flame ionization detector. Ultrapure helium gas (99.999\%, Air Products, UK) was passed through a molecular sieve and oxygen trap (CRS, USA) and was used as a carrier gas with a flow rate of $2 \mathrm{~mL} / \mathrm{min}$. The injection port was held at $250{ }^{\circ} \mathrm{C}$ and operated in the splitless mode for $1 \mathrm{~min}$, then split valve was opened, and a split ratio of 1:5 was applied. Separation was carried out on a DB5, $25 \mathrm{~m} \times 0.32 \mathrm{~mm}$ i.d. and $0.25 \mu \mathrm{m}$ film thickness from SGE (Victoria, Australia) capillary column. The oven temperature was kept at $100{ }^{\circ} \mathrm{C}$ for $2 \mathrm{~min}$, and then increased to $250{ }^{\circ} \mathrm{C}$ at the rate of $10{ }^{\circ} \mathrm{C} / \mathrm{min}$, and was held for $3 \mathrm{~min}$. The FID oven temperature was maintained at $270{ }^{\circ} \mathrm{C}$. Hydrogen was generated by hydrogen generator (OPGU-2200S, Shimadzu) for FID at a flow rate of $40 \mathrm{~mL} / \mathrm{min}$. The flow of air (99.999\%, Air Products) for FID was $400 \mathrm{~mL} / \mathrm{min}$. The model 2010 D centurion scientific centrifuge (Westsussex, UK) was used for separation of sediment phase from sample solution.

Preparation of Magnetic Nanoparticles of $\mathrm{Fe}_{3} \mathrm{O}_{4}$. The chemical co-precipitation method was used in the preparation of the $\mathrm{Fe}_{3} \mathrm{O}_{4}$ nanoparticles (NPs) (26). First, for preparing a stock solution, $10.4 \mathrm{~g} \mathrm{FeCl} \cdot 6 \mathrm{H}_{2} \mathrm{O}$ with $4.0 \mathrm{~g} \mathrm{FeCl}_{2} \cdot 4 \mathrm{H}_{2} \mathrm{O}$ and $1.7 \mathrm{~mL}$ $\mathrm{HCl}(12 \mathrm{~mol} / \mathrm{L})$ were mixed and dissolved in $50 \mathrm{~mL}$ deionized water in a beaker, which was then degassed using nitrogen gas for $20 \mathrm{~min}$ before use. Simultaneously, $500 \mathrm{~mL}$ of $1.5 \mathrm{~mol} / \mathrm{L}$ $\mathrm{NaOH}$ solution was degassed (for $15 \mathrm{~min}$ ) and heated to $80{ }^{\circ} \mathrm{C}$ in a reactor. The stock solution was then added dropwise using a dropping funnel for $30 \mathrm{~min}$ under nitrogen gas protection and with vigorous stirring $(1000 \mathrm{rpm})$ using a glassware stirrer. During the whole process, the solution temperature was maintained at $80{ }^{\circ} \mathrm{C}$, and nitrogen gas was used to prevent the intrusion of oxygen. After the reaction, the obtained $\mathrm{Fe}_{3} \mathrm{O}_{4} \mathrm{NPs}$ precipitate was separated from the reaction medium using magnetic field and then washed four times with $500 \mathrm{~mL}$ of deionized water. Finally, the obtained NPs were resuspended in $500 \mathrm{~mL}$ of degassed deionized water. The obtained NPs were stable under these conditions for up to about one month.

Preparation of the $\mathrm{PPy} / \mathrm{Fe}_{3} \mathrm{O}_{4}$ Nanocomposites. The $\mathrm{PPy}-\mathrm{Fe}_{3} \mathrm{O}_{4}$ nanocomposites were synthesized via in situ polymerization of Py monomer in the presence of $\mathrm{FeCl}_{3}$ as oxidant at an ambient temperature, where suspended $\mathrm{Fe}_{3} \mathrm{O}_{4}$ nanoparticles were encapsulated by the precipitating PPy moieties. In a typical polymerization technique, $0.2 \mathrm{~g} \mathrm{Fe}_{3} \mathrm{O}_{4}$ was added into $25 \mathrm{~mL}$ deionized water in a conical flask and ultrasonicated for 10 min for better dispersion of $\mathrm{Fe}_{3} \mathrm{O}_{4}$ into water. Three grams of $\mathrm{FeCl}_{3}$ oxidant was added into the deionized water containing $\mathrm{Fe}_{3} \mathrm{O}_{4}$ and was shaken for $10 \mathrm{~min}$. To this mixture, $0.8 \mathrm{~mL}$ of polypyrrole was syringed. Then, the reaction mixture was kept under constant shaking for $3 \mathrm{~h}$ at ambient temperature. Finally, to stop the reaction, acetone was added into the reaction mixture. The black powder obtained was filtered, washed with distilled water until the filtrate became colorless, and finally washed with acetone. Then, the composites were dried at $100{ }^{\circ} \mathrm{C}$ for $8 \mathrm{~h}$.

MSPE-DLLME Procedure. $10 \mathrm{~mL}$ of the aqueous sample solution was transferred to a beaker and spiked at the given concentration of the target analytes. $25 \mathrm{mg}$ of $\mathrm{Fe}_{3} \mathrm{O}_{4} @$ PPy NPs was added to the solution and mechanically stirred for $10 \mathrm{~min}$ (Figure 1a). After extraction, the magnetic adsorbents were isolated from the solution with the magnet (Figure 1b). After decanting the supernatant solution, the adsorbed analytes were eluted with $800 \mu \mathrm{L}$ acetonitrile under fierce vortex for $0.5 \mathrm{~min}$ (Figure 1c). Then, the eluent was separated from the NPs with the magnet (Figure 1d). In the next step, $20 \mu \mathrm{L}$ of tetrachloroethylene was added to the eluting solvent of MSPE (Figure 1e). The resulted solution was rapidly injected into $5 \mathrm{~mL}$ water with syringe. A cloudy solution resulting from the dispersion of fine droplets of tetrachloroethylene in the aqueous solution was formed in the test tube with a conical bottom 


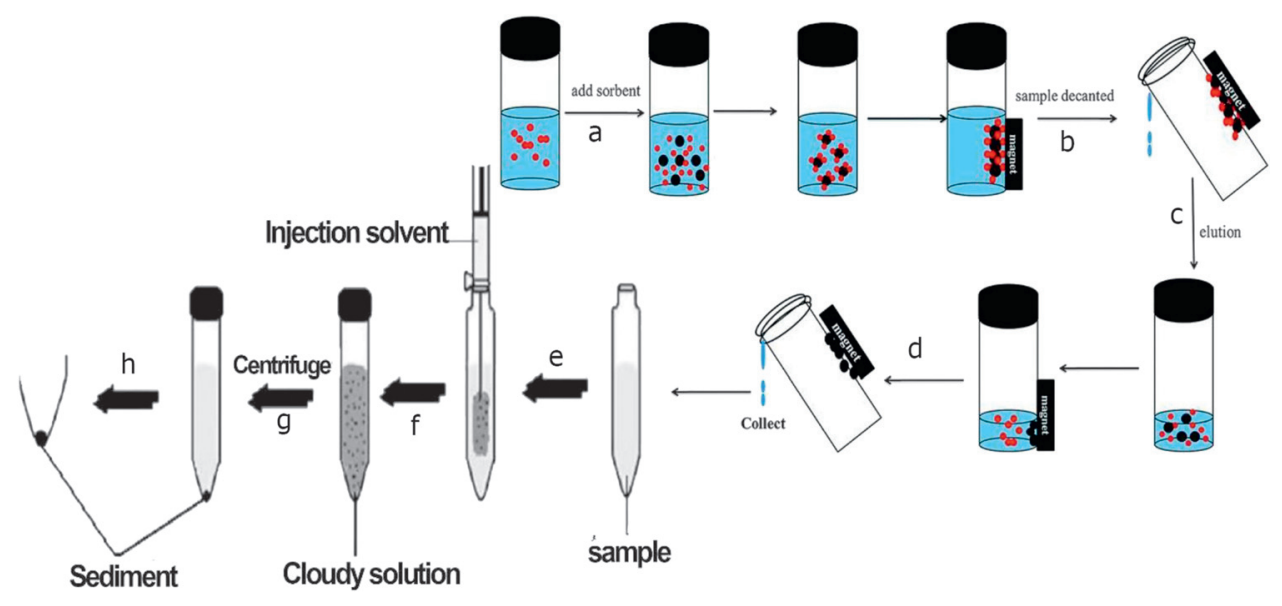

Figure 1. Schematic illustration for the MSPE-DLLME method. NPs was added to the solution and mechanically stirred (a), the magnetic adsorbents were isolated from the solution with the magnet (b), analytes adsorbed were eluted with acetonitrile (c), the eluent was separated from the NPs with the magnet (d), tetrachloroethylene was added to the eluting solvent of MSPE (e), a cloudy solution resulting from the dispersion of fine droplets of tetrachloroethylene in the aqueous solution (f), the solution was centrifuged (g), and the dispersed fine particles of extraction phase settled at the bottom of the conical test tube (h)

(Figure 1f). Then, the solution was centrifuged for $5 \mathrm{~min}$ at $2000 \mathrm{rpm}$ (Figure 1g), and the dispersed fine particles of extraction phase settled at the bottom of the conical test tube (Figure 1h). After that, $2 \mu \mathrm{L}$ of the settled phase was injected into GC-FID for analysis.

\section{Results and Discussion}

In this work, MSPE-DLLME-GC-FID was applied to the simultaneous determination of biphenyl and biphenyl oxide compounds from water samples. To achieve a high extraction recovery $(\mathrm{ER})$ and preconcentration factor $(\mathrm{PF})$, the MSPE and DLLME conditions were optimized. In order to obtain the best extraction performance, different parameters affecting the extraction process, such as sorbent amount, extraction time, elution solvent, volume of elution solvent, type of extraction solvent, volume of extraction solvent, type of disperser solvent, volume of disperser solvent, and desorption time, were studied and optimized. Optimization of the variables mentioned was performed using one-variable-at-a-time method.

Characterization of $\mathrm{Fe}_{3} \mathrm{O}_{4} @$ PPy Nanoparticles. The shape, size, and morphology of the synthesized $\mathrm{Fe}_{3} \mathrm{O}_{4} @$ PPy NPs were determined by transmission electron microscopy (TEM) and scanning electron microscopy (SEM). In addition, the existence and amount of coated PPy were confirmed by Fourier transform infrared (FTIR) spectroscopy. The shape and size of the nanoparticles were observed by TEM (Figure 2a). The TEM images of $\mathrm{Fe}_{3} \mathrm{O}_{4} @$ PPy particles show that an obvious coated

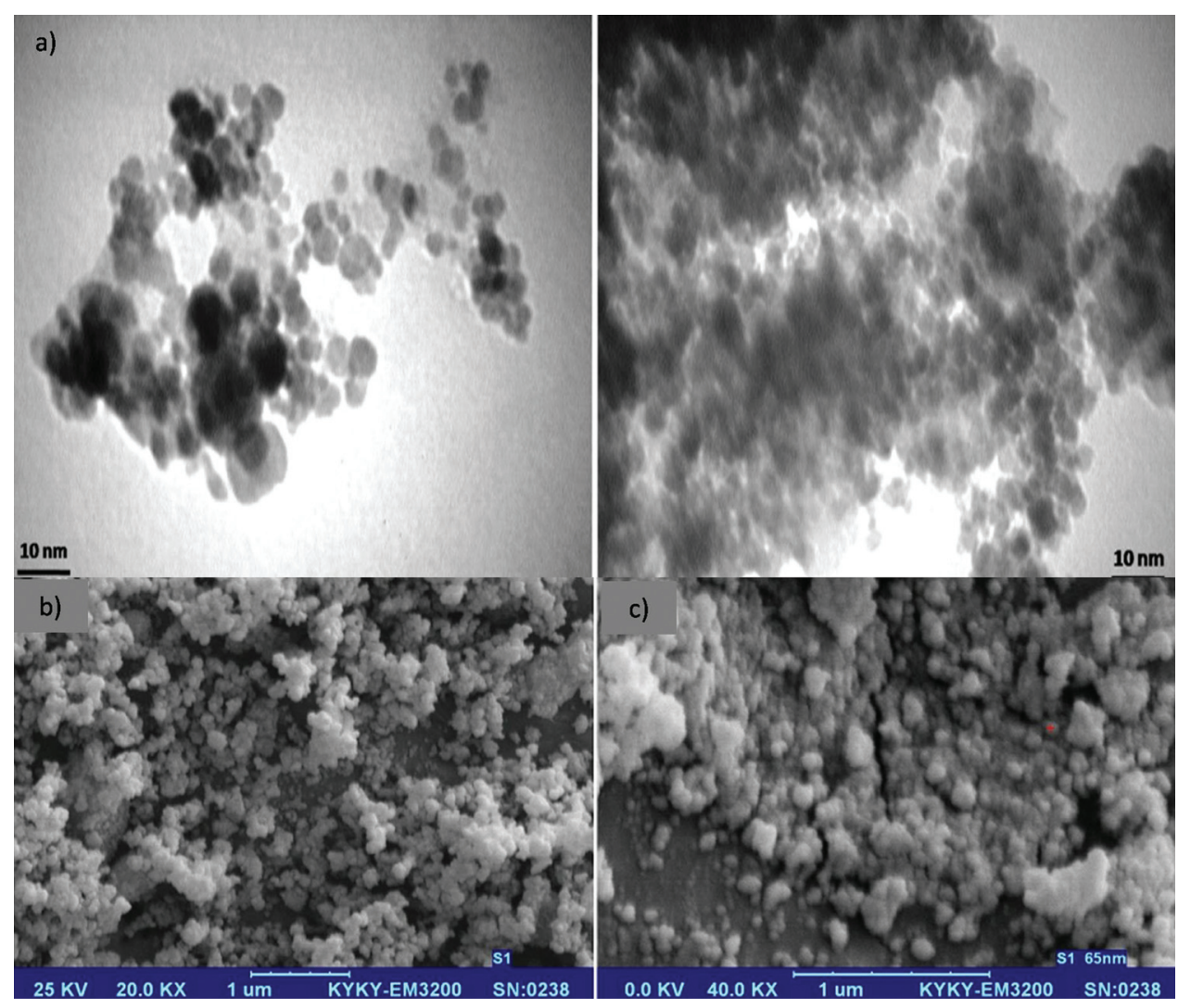

Figure 2. (a) TEM images of $\mathrm{Fe}_{3} \mathrm{O}_{4} @$ PPy. SEM images of $\mathrm{Fe}_{3} \mathrm{O}_{4} @$ PPy NPs: (b) 20,000× and (c) 40,000× magnification 
PPy is immobilized on the surface of $\mathrm{Fe}_{3} \mathrm{O}_{4}$ NPs. The coated PPy layer is clearly seen due to the different electron densities of magnetic nanoparticles core (with dark color) and PPy coating (with light color) in the TEM micrograph. The synthesized $\mathrm{Fe}_{3} \mathrm{O}_{4} @$ PPy NPs showed a spherical shape with an average diameter of about 5-10 nm; however, the nanoparticles tended to aggregate to large particle. According to SEM images (Figure $2 \mathrm{~b}$ and c), the $\mathrm{Fe}_{3} \mathrm{O}_{4} @$ PPy NPs have a nearly spherical shape with a smooth and uniform surface morphology. Due to the agglomeration of the particles and less resolution of SEM as compared to TEM, the size of the particles in the SEM image is larger than that in the TEM image.

The coated PPy was characterized by FTIR in a range of 4000 and $400 \mathrm{~cm}^{-1}$. The FTIR spectra for bare and PPy-coated $\mathrm{Fe}_{3} \mathrm{O}_{4}$ NPs were illustrated in Figure 3. The characteristic absorption peaks of $\mathrm{Fe}_{3} \mathrm{O}_{4} \mathrm{NPs}$, appeared in two spectra (a and b), correspond to the stretching vibrations of hydrogen-bonded surface water molecules and hydroxyl groups at $3400 \mathrm{~cm}^{-1}$, and the $\mathrm{Fe}-\mathrm{O}$ transverse vibration at $580 \mathrm{~cm}^{-1}$ is observable. Coating of PPy onto $\mathrm{Fe}_{3} \mathrm{O}_{4}$ NPs was confirmed by the appearance of characteristic PPy bands in spectrum (b). The weak bands at 2800 and $2900 \mathrm{~cm}^{-1}$ were assigned to the stretching vibrations of $\mathrm{C}-\mathrm{H}$ bonds. The absorption peak at 1050 and $1314 \mathrm{~cm}^{-1}$ were attributed to the bending vibration of $\mathrm{C}-\mathrm{H}$ bond in the pyrrole ring and $\mathrm{C}-\mathrm{N}$ stretching vibration. The absorption bands at 1549 and $1460 \mathrm{~cm}^{-1}$ belong to $\mathrm{C}-\mathrm{C}$ asymmetric and symmetric stretching vibrations of the pyrrole ring, respectively. These results indicate that PPy has been successfully coated on the surface of $\mathrm{Fe}_{3} \mathrm{O}_{4}$ NPs.

Effect of Sorbent Amount. Fewer amounts of nanoadsorbents, due to their greater aspect ratios, might lead to more satisfactory results than micro-adsorbents. To find the optimized amount of adsorbent for the extraction, different amounts of $\mathrm{Fe}_{3} \mathrm{O}_{4} @$ PPy magnetic nanocomposite powder, ranging from 10-40 mg, were examined. Our studies show that the maximum recoveries are achieved when $25 \mathrm{mg}$ of magnetic sorbent was used. When higher amounts of sorbent were used, some of the $\mathrm{Fe}_{3} \mathrm{O}_{4} @$ PPy magnetic nanocomposite might be lost during the collection process by the external field within 1 min duration which leads to a decrease in the recovery values. Therefore, a sorbent amount of magnetic nanocomposite of $25 \mathrm{mg}$ was used for the further experiments.

Effect of Extraction Time. The effect of the extraction time on the extraction recovery of biphenyl and biphenyl oxide was investigated. Due to the shorter diffusion route for NPs and magnetically assisted separation of the MNPs from the sample solutions, extraction can be achieved in less time even for larger volumes of samples. The effect of the extraction time on the extraction recovery was investigated, the findings ranged from 2-20 min. For this work a time duration of $10 \mathrm{~min}$ was sufficient to collect the magnetic sorbent by external magnetic field.

Effect of the Type of Elution Solvent and its Volume. In order to select the most suitable solvent for desorption process, acetonitrile, methanol, ethanol, and acetone were utilized, and the best results were obtained from acetonitrile (Figure 4b).

The volume of acetonitrile was also studied. As depicted in Figure $4 \mathrm{c}$, the best results were obtained from $800 \mu \mathrm{L}$ of acetonitrile. Since the desorption solvent in MSPE step plays the role of dispersive solvent in the DLLME process, further volume increase resulted in decreasing DLLME efficiency due to the raise in solubility of the analytes.

Effect of Type of Extraction Solvent and its Volume. The extraction solvent must be immiscible with water, higher density than water, compatible with the analysis instrument, and have high extraction capability for the analytes. According to these criteria, different organic solvents, such as chloroform, chlorobenzene, tetrachloroethylene, and carbon tetrachloride, were used. The obtained results (Figure 4d) revealed that tetrachloroethylene was the suitable extraction solvent in this method.

In order to study the effect of tetrachloroethylene volume on the extraction performance, different volumes of $\mathrm{C}_{2} \mathrm{Cl}_{4}$ in range of 10-40 $\mu \mathrm{L}$ were investigated. According to the results (Figure $4 \mathrm{e}$ ), $20 \mu \mathrm{L}$ of tetrachloroethylene was chosen as the optimum volume in further work.

Desorption Time. Desorption times were evaluated in the range of $0.5-10 \mathrm{~min}$. The results showed that the time of $0.5 \mathrm{~min}$ is sufficient to quantitative desorption of the analytes.

Sorption Capacity. In order to investigate the sorption capacity of the magnetic sorbent, $10 \mathrm{~mL}$ of aqueous solution containing $1 \mathrm{mg}$ of biphenyl and biphenyl oxide was used. To evaluate the maximum sorption capacity of each analyte, the difference between the concentration of the solution before and after extraction under the optimal condition was calculated. The sorption capacity of nanosorbent, for three parallel experiments, was found to be 21 and $30 \mathrm{mg} / \mathrm{g}$ for biphenyl and biphenyl oxide, respectively.

Quantitative Aspects. As summarized in Table 1, the calibration curve was obtained under the optimized MSPEDLLME-GC-FID conditions. The analytes exhibited good linearity in the range $0.5-100 \mu \mathrm{g} / \mathrm{L}$ with proper correlation coefficients $r^{2}=0.999$ for both of the analytes. The limits of detection (LODs), based on signal-to-noise ratio $(\mathrm{S} / \mathrm{N})$ of 3 , were

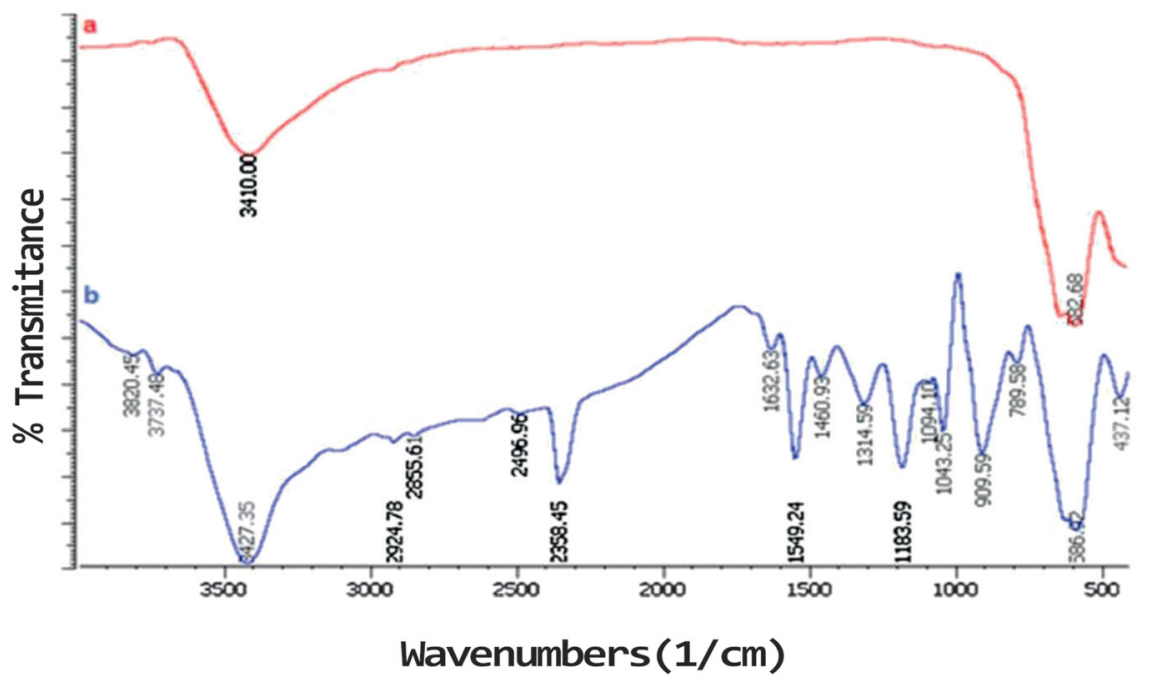

Figure 3. FTIR spectra of (a) $\mathrm{Fe}_{3} \mathrm{O}_{4}$ and (b) $\mathrm{Fe}_{3} \mathrm{O}_{4} @$ aPy 

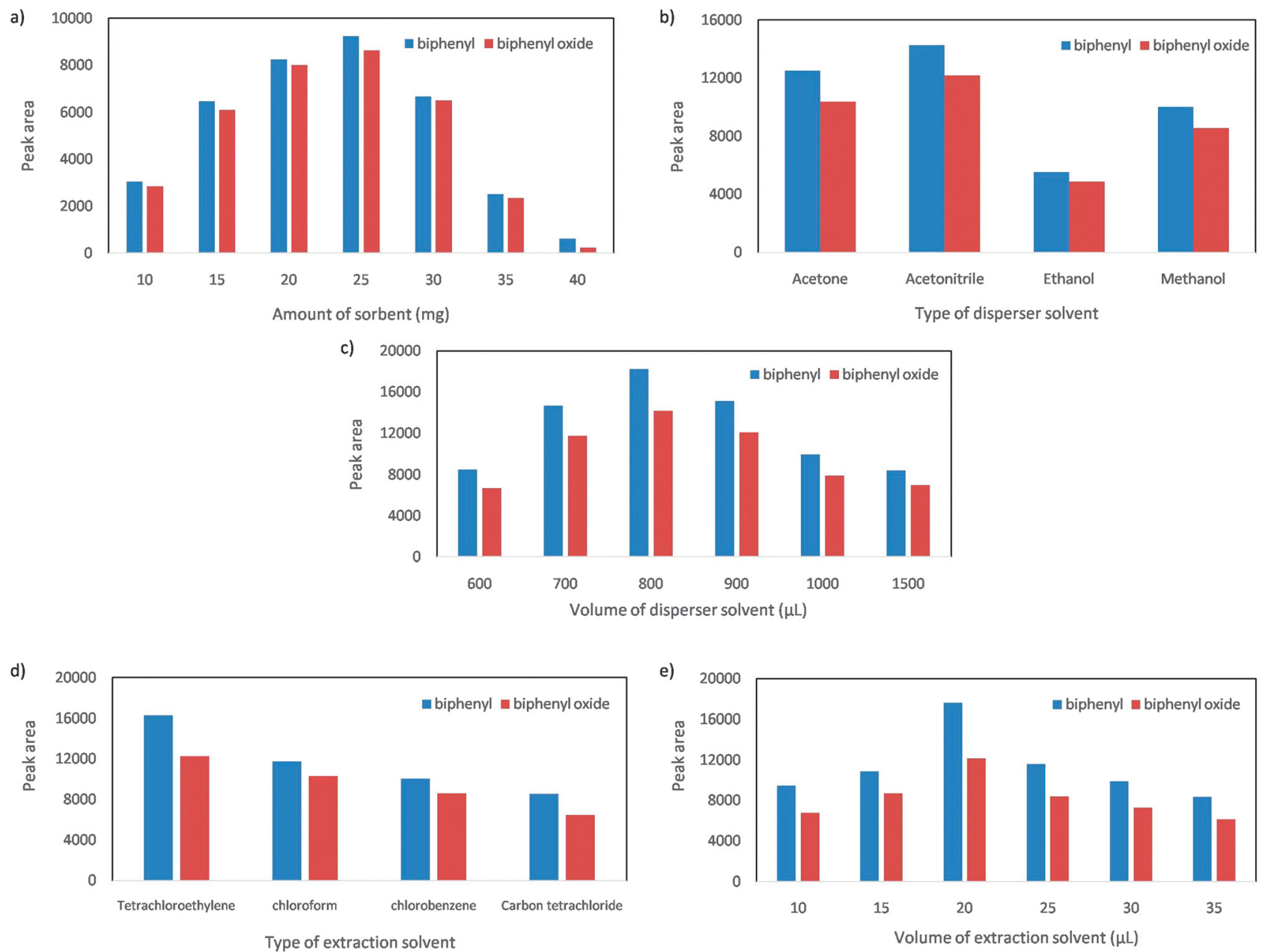

Figure 4. Effect of (a) amount of sorbent, (b) type of disperser solvent, (c) volume of disperser solvent, (d) type of extraction solvent, and (e) volume of extraction solvent on the extraction efficiency of the analytes obtained from MSPE-DLLME. Extraction conditions: desorption time of $30 \mathrm{~s}$, extraction time of $10 \mathrm{~min}$, and sample volume of $10 \mathrm{~mL}$

Table 1. Quantitative results of MSPE-DLLME and GC-FID method for biphenyl and biphenyl oxide

\begin{tabular}{|c|c|c|c|c|c|}
\hline Analyte & Calibration curve equation & Linear range $(\mu \mathrm{g} / \mathrm{L})$ & $\mathrm{LOD}^{a}(\mu \mathrm{g} / \mathrm{L})$ & $\operatorname{RSD}(\%)^{b}$ & $R^{2 c}$ \\
\hline Biphenyl & $Y=29.69 X+58.07$ & $0.5-100$ & 0.03 & 5.1 & 0.999 \\
\hline Biphenyl oxide & $Y=16.83 X+32.61$ & $0.5-100$ & 0.07 & 7.2 & 0.999 \\
\hline
\end{tabular}

0.03 and $0.07 \mu \mathrm{g} / \mathrm{L}$ for biphenyl and biphenyl oxide, respectively. The precision of the method was evaluated by carrying out five independent measurements of the studied compounds at $10.0 \mu \mathrm{g} / \mathrm{L}$. The result showed that the relative standard deviations (RSDs) were 5.1 and $7.2 \%$ for biphenyl and biphenyl oxide, respectively.

Table 2 compares the proposed method with the other extraction methods for the determination of the target analytes in the water samples. The quantitative results of the proposed method are better than those of single drop microextraction (SDME) [1], solid-phase extraction (SPE) [36], and liquid-liquid extraction [37] methods. The comparison of extraction time of the proposed method with that of single drop microextraction
[1] and liquid-liquid extraction [37] for the extraction of the target analytes indicates that this novel method has a very short equilibrium time as compared to the mentioned methods, and the extraction time needed for the proposed method is a few seconds. The quantitative results of the proposed method are comparable with the dispersive liquid-liquid microextraction (SDME) [35]. However, the proposed method has potential for the determination of the target analytes in complex matrices such as waste water because of the MSPE clean-up before DLLME method. Also, it can be used in a large volume of sample, in contrast to the DLLME method. Finally, the proposed method has great potential for determining the selected analytes at the trace levels in water samples.

Table 2. Comparison of the proposed method with other extraction methods for the determination of biphenyl and biphenyl oxide compounds

\begin{tabular}{|c|c|c|c|c|c|}
\hline$\overline{\text { Methods }}$ & RSD (\%) & Dynamic linear range $(\mu \mathrm{g} / \mathrm{L})$ & Limit of detection $(\mu \mathrm{g} / \mathrm{L})$ & Extraction time (min) & Ref. \\
\hline DLLME-GC-FID & $5.5-8.3$ & $0.125-100$ & 0.015 & A few seconds & {$[35]$} \\
\hline SDME-GC-FID & $<5$ & $5-500$ & $1.1-1.8$ & 30 & [1] \\
\hline SPE-GC & $2.1-5.9$ & $50-1500$ & 0.01 & 3 & [36] \\
\hline LLE-GC & $<4$ & $1000-1000000$ & - & 25 & [37] \\
\hline MSPE-DLLME-GC-FID & $5.1-6.7$ & $0.5-100$ & $0.03-0.07$ & 10 & This work \\
\hline
\end{tabular}


a)

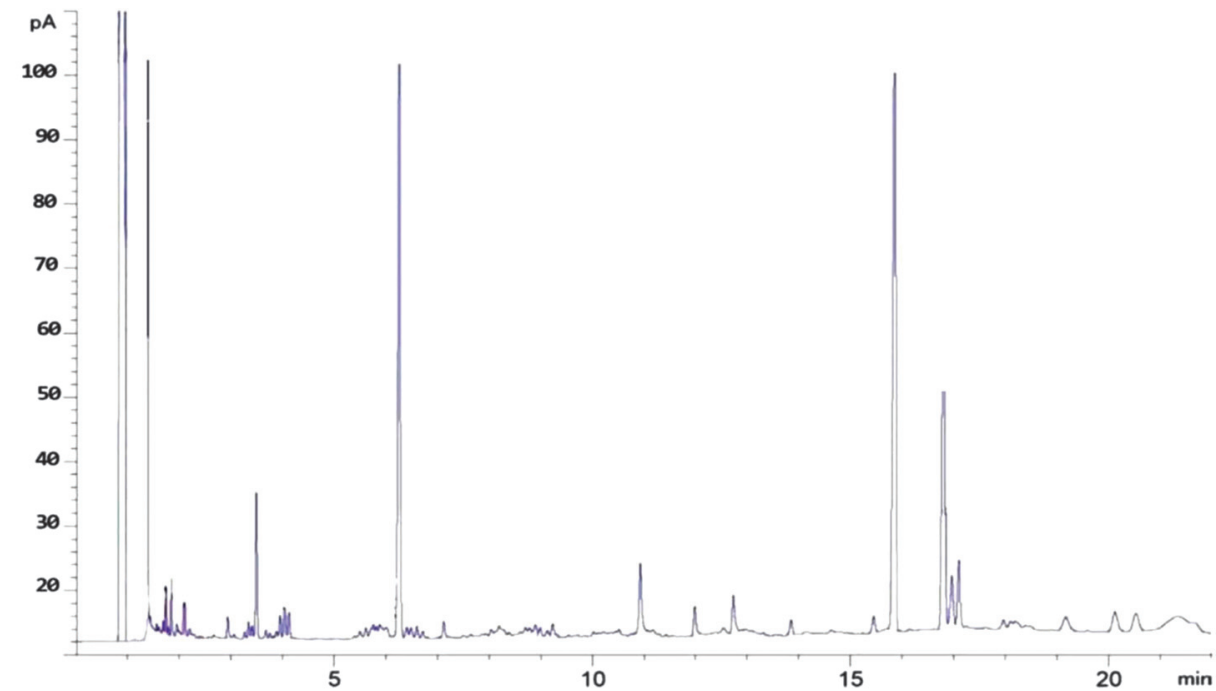

b)

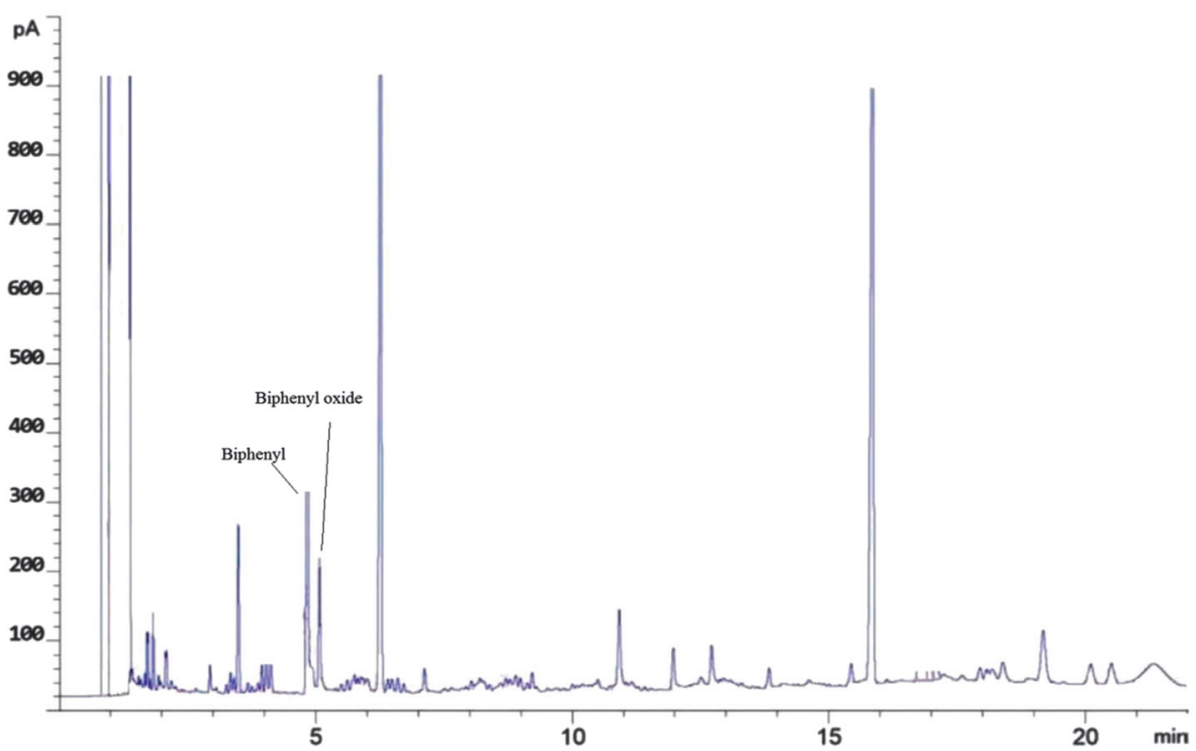

Figure 5. GC-FID chromatograms of the sample (A) before spiking with the analytes in river water and (B) $10.0 \mu \mathrm{g} / \mathrm{L}$ spiked of the analytes in river water after extraction via the proposed method under optimum conditions

Analysis of Real Samples. The proposed MSPE-DLLME technique was applied for the determination of biphenyl and biphenyl oxide compounds in the river water sample to elucidate the applicability and reliability of this method. The water sample was spiked with biphenyl and biphenyl oxide standard solution $(10.0 \mu \mathrm{g} / \mathrm{L}$ concentration level) to assess the matrix effects. The obtained relative recovery was $91 \%$ for biphenyl and $95 \%$ for biphenyl oxide. The results show that matrix has negligible effect on MSPE-DLLME of biphenyl and biphenyl oxide. Figure 5 shows the GC-FID chromatograms of river water prior to (A) and after (B) spiking with biphenyl and biphenyl oxide at $10.0 \mu \mathrm{g} / \mathrm{L}$ level.

\section{Conclusion}

In the present study, combination of polypyrrole-coated $\mathrm{Fe}_{3} \mathrm{O}_{4}$-based MSPE and DLLME was successfully applied as an efficient sample pretreatment method for the trace determination of biphenyl and biphenyl oxide in different water samples. The analytical methodology offers numerous advantages such as ease of operation, high pre-concentration factor, low consumption of organic solvent, and feasibility for large volume samples. Furthermore, the proposed method is promising for the trace analysis of biphenyl and biphenyl oxide in natural water samples.

\section{References}

1. Sarkhosh, M.; Mehdinia, A.; Jabbari, A.; Yamini, Y. Am. J. Anal. Chem. 2011, 2, 689 .

2. American Conference of Governmental Industrial Hygienists (ACGIH), Phenyl ether vapor In: TLVS and other occupational exposure values-1999 [CD-ROM]. Cincinnati OH, USA: ACGIH, 1999.

3. USEPA office of pollution prevention and toxics chemical Facts (accessed, June 1999).

4. http://www.osha.gov, United States Department of Labor, Occupational Safety and Health Administration.

5. Min, D. B.; Wen, J. J. Am. Oil Chem. Soc. 1982, 59, 278

6. EPA Methods 1625-BNW, 1625-S, Semivolatile organic compounds by isotope dilution GC/MS, 1995.

7. Moh, M. H.; Tang, T. S.; Tan, G. H. J. Am. Oil Chem. Soc. 2000, 77, 1077. 8. Asgharinezhad, A. A.; Ebrahimzadeh, H.; Mirbabaei, F.; Mollazadeh, N.; Shekari, N. Anal. Chim. Acta 2014, 844, 80 .

9. Bagheri, H.; Roostaie, A.; Baktash, M. Y. Anal. Chim Acta 2014, 816, 1.

10. Suleiman, J.; Hu, B.; Peng, H. Y.; Huang, C. Z. Talanta 2009, 77, 1579.

11. Wu, Y. W.; Zhang, J.; Liu, J. F.; Deng, Z. L.; Han, M. X.; Jiang, F.; Wang, D. Z.; Wang, H. K.; Yuan, H. Z. Atom. Spectrosc. 2011, 32, 41.

12. Rossi, L. M.; Quach, A. D.; Rosenzwig, Z. Anal. Bioanal. Chem. 2004 380,606 .

13. Xu, H.; Tong, N.; Cui, L.; Lu, Y.; Gu, H. J. Magn. Magn. Mater. 2007, $311,125$.

14. Tahmasebi, E.; Yamini, Y. Anal. Chim. Acta 2012, 756, 13.

15. Galan-Cano, F.; Alcudia-Leon, M. C.; Lucena, R.; Cardenas, S. Valcarcel, M. J. Chromatogr. A 2013, 1300, 134.

16. Lei, Z. I.; Pang, X. L.; Li, N.; Lin, L.; Li, Y. L. J. Mater. Process. Tech. 2009, 209, 3218 .

17. Pu, X. L.; Jiang, Z. C.; Hu, B.; Wang, H. B. J. Anal. At. Spectrom. 2004, 19, 984 . 
18. Camelj, S.; Makovec, D.; Drofenik, M. J. Magn. Magn. Mater. 2009 , $321,1346$.

19. Philippova, O.; Barabanova, A.; Molchanov, V.; Khokhlov, A. Eur. Polym. $J$ 2011, 47,542 .

20. Sing, K. S. Technology profile, Ground Water Monitor 1994, 60.

21. Safarik, I.; Safarikova, M.; Buricova, V. Collect. Czech. Chem. Commun. $1995,60,1448$.

22. Orbell, J. D.; Godhino, L.; Bigger, S. W.; Nguyen, T. M.; Lawrence, N. J. Chem. Educ. 1997, 74, 1446.

23. Jeong, U.; Teng, X.; Wang, Y.; Yang, H.; Xia, Y. Adv. Mater. 2007, 19, 33. 24. Bagheri, H.; Saraji, M. J. Chromatogr. A 2003, 986, 111.

25. Bagheri, H.; Mahammadi, A.; Salemi, A. Anal. Chim. Acta 2004 513,445 .

26. Wu, T. M.; Lin, S. H. J. Polym. Sci. Part A: Polym. Chem. 2006, 44, 6449.

27. Han, G.; Yuan, J.; Shi, G.; Wei, F. Thin Solid Films 2005, 474, 64.
28. Wu, J.; Lord, H. L.; Pawliszyn, J.; Kataoka, H. J. Microcolumn Sep. 2000, 12,255

29. Vickackaite, V.; Ciuvasovaite, V. Cent. Eur J. Chem. 2007, 5, 727.

30. Meng, J.; Bu, J.; Deng, C.; Zhang, X. J. Chromatogr. A 2011, 1218, 1585.

31. Rezaee, M.; Assadi, Y.; Milani Hosseini, M. R.; Aghaee, E.; Ahmadi, F.; Berijani, S. J. Chromatogr. A 2006, 1116, 1.

32. Rezaee, M.; Yamini, Y.; Shariati, S.; Esrafili, A.; Shamsipur, M. J. Chromatogr. A 2009, 1216, 1511.

33. Rezaee, M.; Yamini, Y.; Faraji, M. J. Chromatogr. A 2010, 1217, 2342.

34. Mashayekhi, H. A.; Abroomand-Azar, P.; Saber-Tehrani, M.; Waqif, S. H. Chromatographia 2010, 71, 517.

35. Mashayekhi, H. A.; Abroomand-Azar, P.; Saber-Tehrani, M.; Waqif, S. H. Int. J. Environ. Anal. Chem. 2011, 91, 516.

36. Yamazaki, Y.; Niomiya, T. J. AOAC Int. 1999, 82, 1474

37. Westoo, G.; Andersson, A. Analyst 1975, 100, 173. 